\title{
Performance of small group majorities in a single task
}

\author{
Henrik Olsson *
}

\begin{abstract}
Aggregating decisions from larger groups typically results in outcomes with higher accuracy than decision outcomes from single individuals or smaller groups. Here I argue that it is important to consider not only overall proportion of correct decisions, but also individual competencies in terms of hits $(h)$ and correct rejections $(c r)$. I show that small groups can perform better than randomly selected individuals and larger groups in a single task when the average individual proportion correct is above $.5, h$ and $c r$ are asymmetric around .5 , and $h+c r>1$. If the average individual proportion correct is below .5 and $h+c r<1$, small groups perform worse than individuals and larger groups. I also demonstrate that these two performance patterns can occur in empirical data from studies on violent recidivism, psychiatric morbidity, anxiety, and deception detection. I also show that the presence of correlations between decisions in a single task has both beneficial and detrimental effects when it comes to small group performance.
\end{abstract}

Keywords: Condorcet jury theorem, wisdom of crowds, hits, correct rejections

\section{Introduction}

It is typically assumed that aggregating decisions from larger groups improves accuracy compared to decisions of a single individual or from a smaller group. Can a smaller group ever outperform a larger group? Condorcet's Jury Theorem (CJT, Condorcet, 1785) provides one argument for the supremacy of larger groups, a phenomenon that is commonly referred to as the wisdom of crowds (Page, 2007; Sunstein, 2006; Surowiecki, 2004). The CJT states that if the individual competence, or accuracy, of independent group members is larger than .5, a majority vote in larger groups will be more accurate than in smaller groups and the probability that the majority vote is correct will approach 1.0 as the number of voters increases. This is the basis for the findings that accuracy increases with expert group size in a variety of settings (see e.g., Kurvers, de Zoete, Bachman, Algra, \& Ostelo, 2018, for an example in the medical domain).

There are, however, situations where a small group can outperform individuals and larger groups. If the individuals are selected according to past performance, smaller groups can outperform larger groups (Budescu \& Chen, 2015; Mannes, Soll, \& Larrick, 2014; Olsson \& Loveday, 2015). Even when individuals are randomly selected from a larger group of people, there are situations where smaller groups can outperform larger ones. If the group encounters tasks of different difficulties, Galesic, Barkoczi, and Katsikopoulos (2018) showed that smaller groups can achieve higher accuracy on average across tasks than either individ-

\footnotetext{
I thank Mirta Galesic for helpful comments on an earlier version of this paper.

*Santa Fe Institute, 1399 Hyde Park Rd. Santa Fe, NM 87501. Email: olsson@santafe.edu.
}

uals or larger groups.

I show that small randomly selected groups can outperform individuals and large groups even in a single task. A small-group advantage in terms of overall proportion correct $P$ can be observed for a single task if the individual probability of hits ( $h$, saying that the state of the world is 1 when it is indeed 1) and individual probability of correct rejections ( $c r$, saying the state of the world is 0 when it is indeed 0 ; or equivalently 1 minus the probability of false alarms, $f a$ ) are not the same, as is assumed in the traditional formulation of the CJT. Specifically, a small-group advantage can be observed when the group proportion correct $P$ is larger than .5 , the individual competencies are asymmetric around .5 (i.e., $h$ and $c r$ are on the opposite sides of .5 and not equidistant from .5) and $h+c r>1$ (lower case letters refer to individual performance, while uppercase letters refer to group performance). I will 1) describe four cases relating group size and accuracy and the conditions under which they occur, 2) show four empirical demonstrations from research on violent recidivism, psychiatric morbidity, anxiety, and deception detection, and 3) illustrate the effects of correlated decisions on the four cases relating group size and accuracy, as modeled with a leader-follower model (Boland, Proschan, \& Tong, 1989; Spiekermann \& Goodin, 2012).

\section{Small-group advantage and disad- vantage in a single task}

In most discussions of CJT the definition of accuracy as proportion correct implicitly assumes that the accuracy when the event occurs is the same as the accuracy when the event does not occur. In the language of signal detection theory 
(Macmillan \& Creelman, 2005; Sorkin, Hays, \& West, 2001), this means that the individual probability of hits, $h$, and the individual probability of correct rejections, $\mathrm{cr}$ are the same. This assumption is problematic from policy and decision theoretic perspectives, where different outcomes can have different pay-offs or utilities. In a legal setting where a jury is to decide on a guilty or not guilty verdict, there might be a presumption that the legal system should be biased towards maximizing a jury's $C R$ (or equivalently, minimizing $F A$, i.e., avoiding convicting innocent suspects). Juries might therefore be encouraged to achieve a group decision high $C R$, even if it comes at the expense of low $H$.

Empirical asymmetries between average individual levels of hits, $h$, and correct rejections $c r$, have indeed been observed in many real-world situations. In any decision situation where the decision criterion can be varied to obtain different $h$ and $c r$, situations can occur where $h$ is above .5 and the $c r$ is below .5. In other situations, $h$ can be found to be below .5 and $c r$ above .5. For example, for the case of predicting violent recidivism Rice and Harris (1995) found that for low base rates $(.15)$ of violent recidivism $h(.35)$ was much lower than $\operatorname{cr}(.88$, or equivalently a $f a$ of .12$)$.

Assuming that $H$ and $C R$ are based on independent Bernoulli trials as in the CJT, the relation between $H, C R$ and $P$ for a certain group size $n$, can be expressed as:

$$
P_{n}=b r H_{n}+(1-b r) C R_{n}
$$

where $P_{n}$ is the average accuracy for a group of size $n$, $b r$ is the base rate of the event, $H_{n}$ is the hit rate for the majority decision made by a group of size $n$, and $C R_{n}$ is the correct rejection rate for the majority decision made by a group of size $n . H_{n}$ and $C R_{n}$ are determined by the binomial cumulative distribution function with parameters $n, h$ and $c r$. The values of $h$ and $c r$ are assumed to be the averages of the individual values.

The convergence properties for $P$ expressed as a function of $H$ and $C R$ are dependent upon the values of the individual competencies, $h$ and $c r$ (throughout the paper I assume homogeneous individual competencies, hence the omission of the bar over $h$ and $c r$ ). As $n$ grows large $P$ converges to:

$$
P=\left\{\begin{array}{cll}
1 & \text { if } & h>.5 \text { and } c r>.5 \\
0 & \text { if } & h<.5 \text { and } c r<.5 \\
b r & \text { if } & h>.5 \text { and } c r<.5 \text {, as } n \rightarrow \infty \\
1-b r & \text { if } & h<.5 \text { and } c r>.5 \\
.5 & \text { if } & h=.5 \text { and } c r=.5
\end{array}\right.
$$

When both $h$ and $c r$ are on the same side of .5 , the results are analogues to CJT results: $P$ converges to 1 when $h>.5$ and $c r>.5$ (analogous to the case in CJT where the average of individual competencies, $\bar{p}$, is above .5) and converges to 0 when $h<.5$ and $c r<.5$ (analogous to the case in CJT where the average of the individual competencies, $\bar{p}$, is below .5). When $h$ and $c r$ are on the opposite sides of .5 , however, $P$ converges to either the base rate, $b r$, or to $1-b r$. The reason for the convergence to the $b r$ is that when $n$ becomes large and $h>.5, H$ converges to 1 , and when $\mathrm{cr}<.5, C R$ converges to 0 , leaving the base rate of the event: $b r \cdot 1+(1-b r) \cdot 0=b r$. When $h<.5$, on the other hand, $H$ converges to 0 , and when $c r>.5, C R$ converges to 1 , leaving one minus the base rate of the event: $b r \cdot 0+(1-b r) \cdot 1=1-b r$. Figure 1 shows simulation results for $H, C R$, and $P$ as a function of group size for the first four cases in Equation 2. The black circles in Figure 1 indicate the asymptotic value for $P$ when $n=10,001$ and $b r=.6$.

Next, I discuss four cases relating group size and accuracy: group advantage, individual advantage, small-group advantage, and small-group disadvantage. I define largegroup advantage as $P_{n+2}>P_{n}$ for all $n$. An individual advantage is defined as $P_{n}>P_{n+2}$ for all $n$. A small-group advantage occurs when there exists a group size $s$ for which $P_{s}>P_{n \leq s-2}$ and $P_{s}>P_{n \geq s+2}$, for all $n \neq s$. A small-group disadvantage is defined as having a global proportion correct minimum for a group of size $n$, with $P_{s}<P_{1}$ (where $\left.P_{1}=\bar{p}\right)$, and $P_{s}<P_{n}$.

The conditions for when the small-group advantage occurs follow the same logic as their occurrence when there is a mix of easy and difficult tasks (Galesic et al., 2018). When $h>.5$ and $c r<.5$ or when $h<.5$ and $c r>.5$, a smallgroup advantage is observed when $h+c r>1$ and $P_{1}$ is larger than .5 (as calculated by eq. 1). Conversely, a small-group disadvantage is observed when $h+c r<1$ and $P_{1}$ is smaller than .5. I should note that there are cases when the smallgroup effects do not occur in these conditions. Specifically, when $P_{1}$ is close to .5 the expected group size for the smallgroup advantage will be in the region of $1 \leq n \leq 3$ and therefore sometimes undetectable.

\section{Empirical demonstrations}

In wisdom of crowd studies it is typically assumed, and empirically confirmed, that increasing group size will lead to higher accuracy, at least for non-interacting individuals with less dependent decisions. For example, studies on the accuracy of physician's detection performance of various conditions such as breast or skin cancer (e.g., Kurvers et al., 2016) almost invariably show a monotonically increasing accuracy with group size (e.g., Kurvers et al., 2018). These studies, however, rely mostly on accepted tests and criteria that have average individual hits and correct rejections well above .5. There are, however, other predictive and diagnostic situations where the average individual hits and correct rejections are on the opposite sides of .5. Figure 2 shows four examples: violent recidivism $(h=.37, c r=.88, b r=.15$; Rice \& Harris, 1995), physician's detection of psychiatric morbid- 

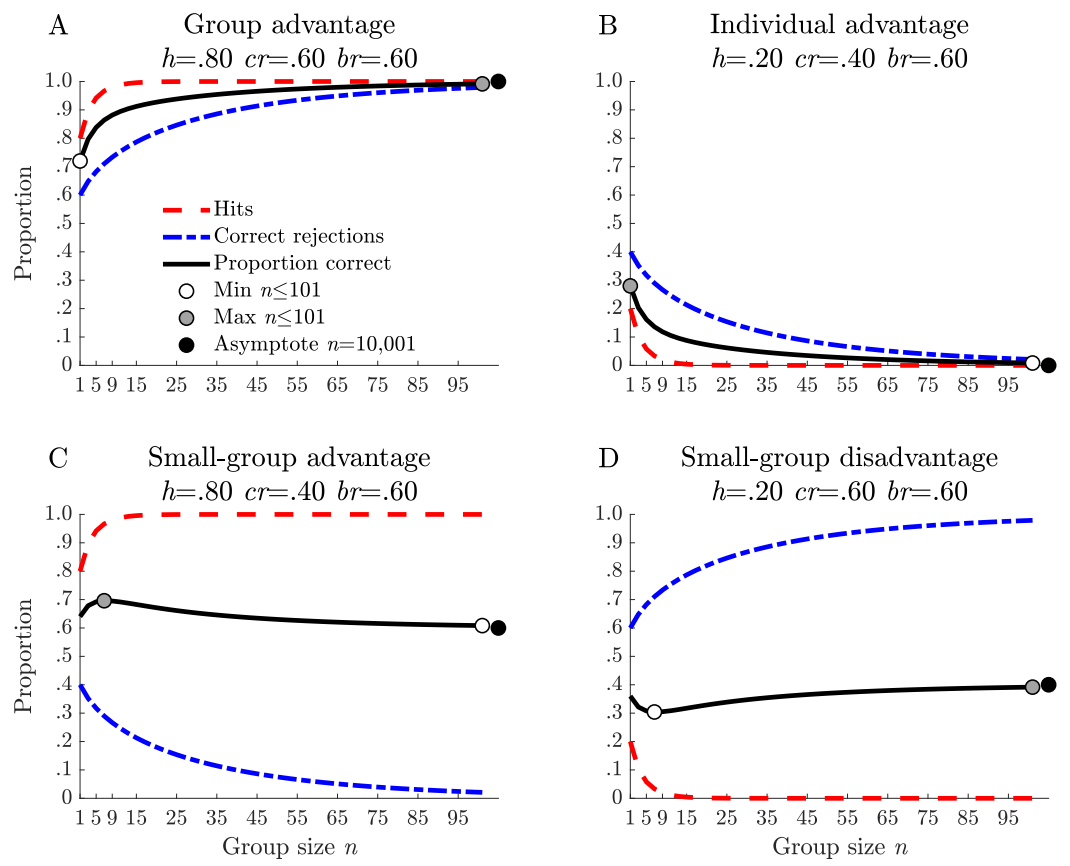

Figure 1: Simulation results for $H, C R$, and $P$ as a function of group size $n$ for examples of the first four cases in Equation 2. White circles are the minimum values of $P_{n}$ for $n \leq 101$. Gray circles are the maximum values of $P_{n}$ for $n \leq 101$. Black circles are the asymptotic values of $P_{n}$ for $n=10,001$.
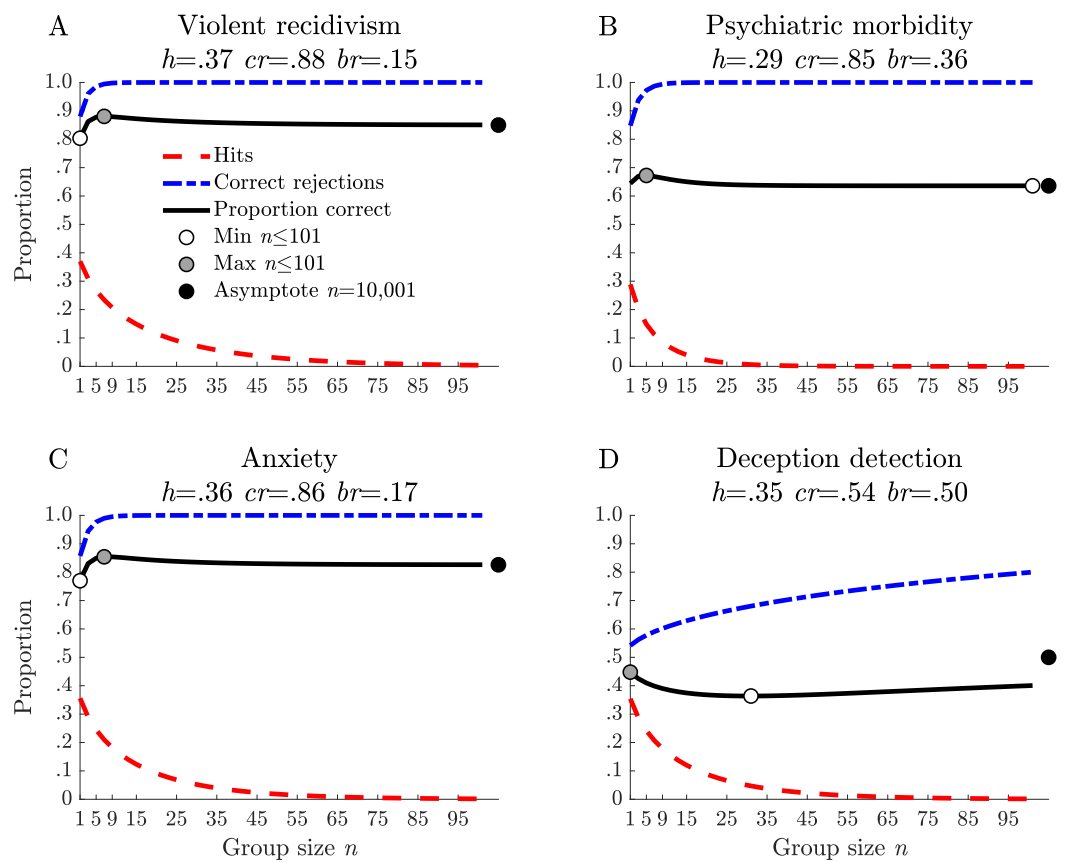

Figure 2: Simulation results based on empirical $H, C R$, and $P$ as a function of group size $n$. White circles are the minimum values of $P_{n}$ for $n \leq 101$. Gray circles are the maximum values of $P_{n}$ for $n \leq 101$. Black circles are the asymptotic values of $P_{n}$ for $n=10,001$. 
ity among cancer patients $(h=.29, \mathrm{cr}=.85, \mathrm{br}=.36$; Fallowfield, Ratcliffe, Jenkins, \& Saul, 2001), physician's detection of anxiety among dermatology patients $(h=.36$, $c r=.86, b r=.17$; Dalgard et al., 2018), and lie detection in a laboratory setting $(h=.35, c r=.54, b r=.50$; Reinhard, Greifeneder, \& Scharmach, 2013). The first three examples have $h+c r>1$ and therefore a small-group advantage is observed for these cases. The lie detection example, however, has $h+c r<1$ and a small-group disadvantage is observed.

\section{Leader-follower convergences}

So far, I have assumed that individual decisions are independent from each other. However, in many real-world situations this does not hold. Therefore, it is essential to also understand the effects of correlations on the accuracy of group decisions. I implemented dependencies among individual decisions with a leader-follower model (Boland et al., 1989; Galesic et al., 2018; Kao \& Couzin, 2014; Spiekermann \& Goodin, 2012). The leader-follower model has a parameter $r$ that determines the proportion of group members that follow the leader, effectively controlling the correlations between group members. I implemented the leader-follower model as an extension of Equation 1 with expressions that allow for the combined effects of $H_{n}$ (here depending on $h$ and $r$ ), $h, C R_{n}$ (here depending on $c r$ and $r$ ), $c r$, leader hits $h_{l}$, leader correct rejections $c r_{l}$, and $b r$. First, define the probabilities in the cumulative binomial distribution as $h^{\prime}=h(1-r)+r, h^{\prime \prime}=h(1-r), c r^{\prime}=c r(1-r)+r$, and $c r^{\prime \prime}=c r(1-r)$. Then, define $H_{n, h}, H_{n, h \prime \prime}, C R_{n, c r \prime}$, and $C R_{n, c r \prime \prime}$ as the corresponding values of the group hit rates and correct rejections for $n$ and $r$. The proportion correct for a certain group size is then:

$$
\begin{aligned}
P_{n} & =b r\left[h_{l} H_{n, h \prime}+\left(1-h_{l}\right) H_{n, h_{\prime \prime}}\right] \\
& +(1-b r)\left[c r_{l} C R_{n, c r}+\left(1-c r_{l}\right) C R_{n, c r \prime \prime}\right]
\end{aligned}
$$

Extensive computer simulations showed that across the whole range of $r$, the superiority of moderate group sizes still holds (not shown).

Here I go further and provide convergence properties for overall proportion correct $\bar{P}$, assuming equal proportion of hits and correct rejections for the leader and the group members. Convergence results can be derived by noting that for a single task in the leader-follower model, $P$ converges to $p_{l}$ if $r>(p-0.5) / p$ (Goodin \& Spiekermann, 2012). First, simplify the right side of the inequality to $1-(0.5 / p)$. Then, substitute $p$ with the minimum or maximum value of $h$ and $c r$ or their complements $h^{\prime}=1-h$ and $c r^{\prime}=1-c r$ (contrary to $p$ in the previous inequality, $h$ and $c r$ range between zero and one). The convergence properties are summarized in Table 1.
Thus, $P$ will converge to $p_{l}, 0,1, b r$, or $1-b r$ depending on the values of $h, c r$, and $r$. If the values of $r$ are between the boundaries specified in Table 1 , the convergence of $P$ can be calculated by using asymptotic values in Equation 3. For example, if the term $H_{n, h}$, is larger than .5 it will converge to 1 and if the term is below .5 it will converge to 0 .

Figures 3 and 4 illustrate the effects of $r$ for the same four cases shown in Figure 1. In all panels of Figure 3 the value of $r$ was set to be lower than .167 , which is the lower boundary of $r$ for all combinations of $h$ and $r$ in the different panels. In all panels of Figure 4, the value of $r$ was set to be larger than .375 , which is the upper boundary of $r$ for all combinations of $h$ and $r$ in the different panels. The average proportions correct $p_{l}=\bar{p}$ were $.72, .28, .64$, and .36 (calculated with Equation 2) for the four different combinations of $b r, h$ and cr.

Figure 3 shows that a value of $r$ of .15 has little effect on proportion correct $\mathrm{P}$ compared to the case with independent decisions illustrated in Figure 1: The advantages and disadvantages show the same patterns, although the convergence rates are lower. The one difference is that in Figure 3D, there are both an individual advantage and a large group advantage as there are two maxima at $n=1$ and when $n$ goes to infinity (i.e., $P_{1}$ and the leader's proportion correct, $p_{l}$, are the same). When $r=.5$, the patterns change. In Figure 4A we observe a small group advantage, while Figures $1 \mathrm{~A}$ and 3A show a large group advantage. Figure 4B shows a small group disadvantage, while Figures $1 \mathrm{~B}$ and $3 \mathrm{~B}$ show an individual advantage. Figure $4 \mathrm{C}$ shows a small group advantage, as do Figures $1 \mathrm{C}$ and $3 \mathrm{C}$. Figure 4D shows a small group disadvantage, the same as in Figures 1D and 3D.

\section{Discussion}

In this paper I showed that a small-group advantage can be achieved for a single task, given that the average proportion correct for a single individual is above .5, the individual competencies are asymmetric around .5 (i.e., $h$ and $c r$ are on the opposite sides of .5 and not equidistant from .5) and $h+c r>1$. If $h+c r<1$ a small-group disadvantage is observed. The empirical demonstrations showed that these patterns can occur in real-world decision situations. Finally, I showed that introducing correlations between decisions can have both detrimental and beneficial effects when it comes to a small-group advantage.

The results on this paper show that When planning for the size of committees for real-world decisions, it is important to consider not only overall proportion correct, but also hits, correct rejections, and the degree of correlation between decisions. For example experts deciding if a convicted criminal is likely to reoffend can have low hit rates and high correct rejection rates (Rice \& Harris, 1995). In situations such as these, a small group of experts might outperform a larger one 
TABLE 1: Leader-Follower convergences as $n \rightarrow \infty . h^{\prime}=1-h$ and $c r^{\prime}=1-c r$.

\begin{tabular}{l} 
if $h>.5$ and $c r>.5:$ if $r<1-\frac{0.5}{\min (h, c r)}$ then $P=1, \quad$ if $r>1-\frac{0.5}{\max (h, c r)}$ then $P=p_{l}$ \\
if $h<.5$ and $c r<.5:$ if $r<1-\frac{0.5}{\min \left(h^{\prime}, c r^{\prime}\right)}$ then $P=0, \quad$ if $r>1-\frac{0.5}{\max \left(h^{\prime}, c r^{\prime}\right)}$ then $P=p_{l}$ \\
if $h>.5$ and $c r<.5:$ if $r<1-\frac{0.5}{\min \left(h, c r^{\prime}\right)}$ then $P=b r, \quad$ if $r>1-\frac{0.5}{\max \left(h, c r^{\prime}\right)}$ then $P=p_{l}$ \\
if $h<.5$ and $c r>.5:$ if $r<1-\frac{0.5}{\min \left(h^{\prime}, c r\right)}$ then $P=1-b r$, if $r>1-\frac{0.5}{\max \left(h^{\prime}, c r\right)}$ then $P=p_{l}$ \\
\hline
\end{tabular}

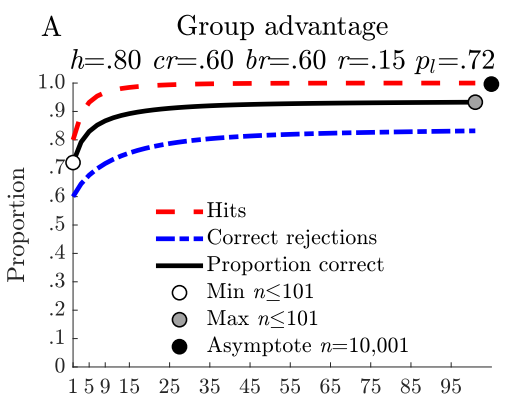

B Individual advantage
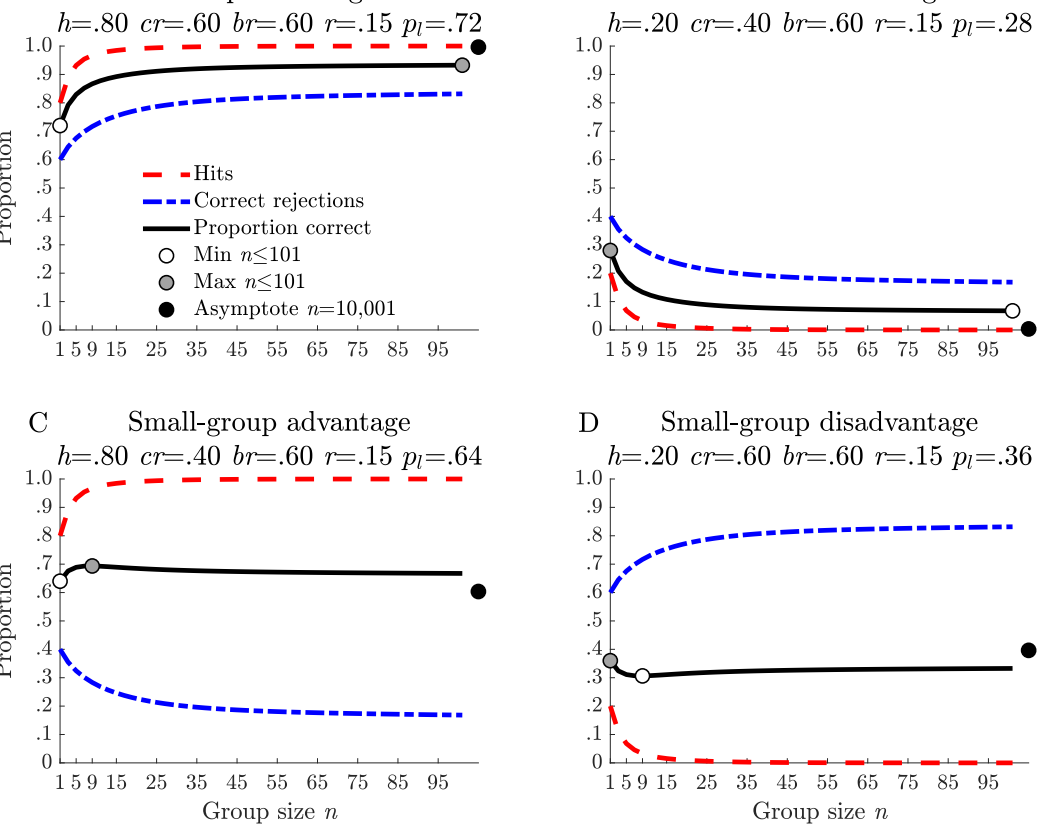

Figure 3: Effects of $r=.15$ on $P$ for the same values of $h, c r$, and $b r$ as in Figure 1. $p_{l}$ is the leader's proportion correct. White circles are the minimum values of $P_{n}$ for $n \leq 101$. Gray circles are the maximum values of $P_{n}$ for $n \leq 101$. Black circles are the asymptotic values of $P_{n}$ for $n=10,001$.

(see Figure 2A, 2B, 2C). However, if $h+c r<1$ a small group of experts is less accurate than a randomly chosen expert and a larger group of experts (See Figure 2D). More generally, past information about the pattern of hits and correct rejections can give clues as to what is to be expected: large-group advantage, individual advantage, small-group advantage, or small-group disadvantage.

My results also show the importance of dependencies among individual decisions. A high correlation can produce a small-group advantage even when hits and correct rejections are both above .5 , but this same correlation diminishes the small-group advantage found when hits and correct rejections are asymmetric around .5. In some real-world decision situations, such as jury deliberations, it might not be the case that the hits and correct rejections which are asymmetric around .5 will produce a small-group advantage. The nature of the decision situation would nevertheless be conducive to such an effect as the information sharing among individuals would result in dependencies among individuals which can produce a small-group advantage even when the hits and correct rejections are above .5. As with decision situations without dependencies among individuals, knowing the hits, correct rejections, and the level of dependencies can give clues as to what is to be expected in terms of accuracy when group size increases and how to best determine the size of a committee. For example, the boosting of a small-group advantage when there are high correlations among individual decisions might provide an additional mechanism that can help explain contradictory findings of small or negative effects of group size on accuracy in interacting groups.

In this paper I focused on proportion correct, hits, and false alarms. There are many other measures of accuracy such as positive predictive value, negative predictive value, positive likelihood ratio, diagnostic odds ratio and so on (for an overview, see Singh, 2013). It would be worthwhile to investigate the prevalence of large-group advantage, individual advantage, small-group advantage, or small-group disadvantage for these measures too. However, if the mea- 

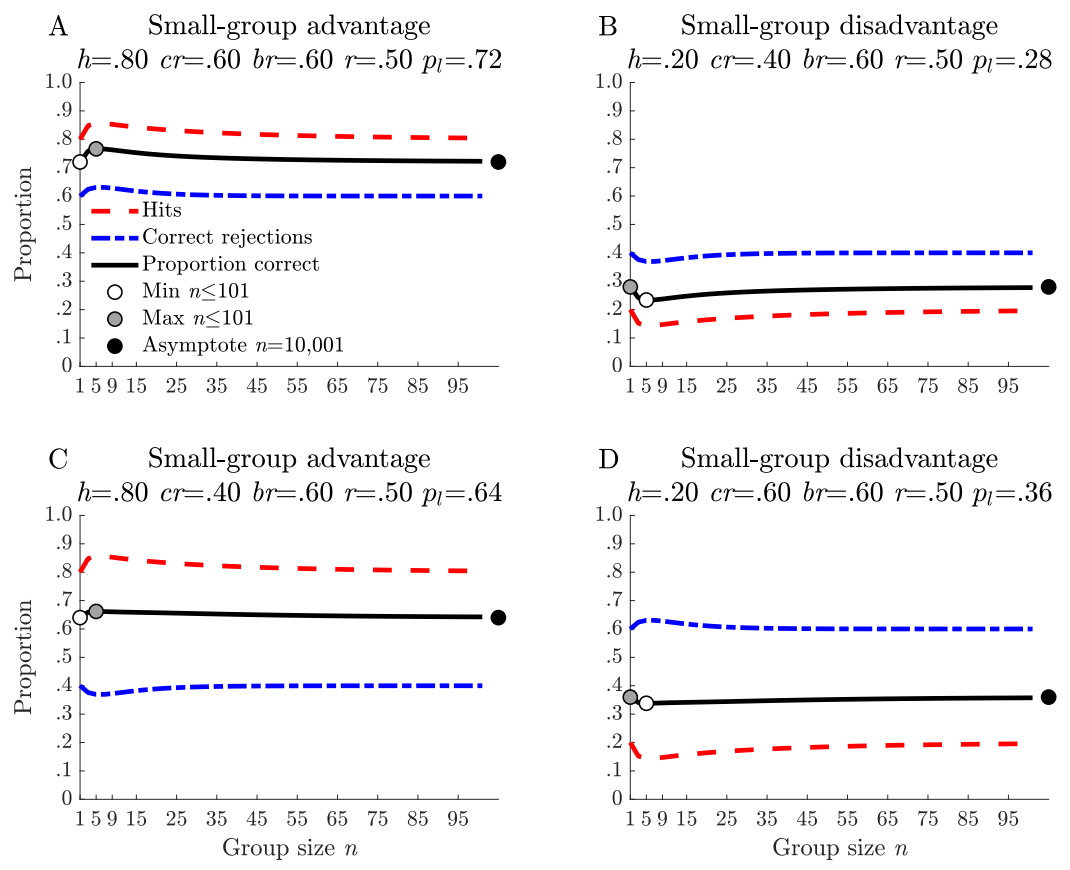

Figure 4: Effects of $r=.5$ on $P$ for the same values of $h, c r$, and $b r$ as in Figure 1. $p_{l}$ is the leader's proportion correct. White circles are the minimum values of $P_{n}$ for $n \leq 101$. Gray circles are the maximum values of $P_{n}$ for $n \leq 101$. Black circles are the asymptotic values of $P_{n}$ for $n=10,001$.

sures include components that are monotonically related to hits and false alarms, for example, the diagnostic odds ratio one would expect that such measures also would exhibit similar patterns as proportion correct with asymmetric hits and correct rejections.

The demonstrations in this paper used the binomial distribution function, assumed equal competencies across individuals, and implemented dependencies between individuals in a simple leader-follower model. All these assumptions and model choices in the simulations can be challenged. One crucial assumption that needs to be further investigated is different assumptions made about the dependencies among individuals. For example, in the leader-follower model used here, the parameter $r$ can never cause the correlations among individuals to be negative and the competencies for the leader were the same as for the individuals in the group.

The results in this paper are only applicable to categorical decisions. If continuous judgments are aggregated and the resulting group error is measured with mean squared error (or any convex loss function), a larger group will always have smaller error than the groups average individual. This follows from what has been called the "averaging principle" (Larrick \& Soll, 2006), the "diversity prediction theorem" (Page, 2007) or the "ambiguity decomposition" (Krogh \& Vedelsby, 1995): the error of an aggregate estimate is equal to the difference between average individual error and the variability of the individual estimates around the aggregate estimate, or their diversity. In other words, for continuous judgments the group will be better than the average individual as long as there is some diversity in the individual estimates. As I show here, this is not always the case for categorical decisions.

\section{References}

Boland, P. J., Proschan, F., \& Tong, Y. L. (1989). Modelling dependence in simple and indirect majority systems. Journal of Applied Probability, 26(1), 81-88. doi: $10.2307 / 3214318$

Budescu, D. V., \& Chen, E. (2015, may). Identifying expertise to extract the wisdom of crowds. Management Science, 61(2), 267-280. doi: 10.1287/mnsc.2014.1909

Condorcet, M. (1785). Essai sur l'application de l'analyse à la probabilité des décisions rendues à la pluralité des voix [Essay on the application of analysis to the probability of majority decisions]. Paris, France: Imprimerie Royale.

Dalgard, F. J., Svensson, Å., Gieler, U., Tomas-Aragones, L., Lien, L., Poot, F., .. Kupfer, J. (2018, may). Dermatologists across Europe underestimate depression and anxiety: Results from 3635 dermatological consultations. British Journal of Dermatology, 12(10), 3218-3221. doi: 10.1111/bjd.16250

Fallowfield, L., Ratcliffe, D., Jenkins, V., \& Saul, J. (2001). 
Psychiatric morbidity and its recognition by doctors in patients with cancer. British Journal of Cancer, 84(8), 1011-1015. doi: 10.1054/bjoc.2001.1724

Galesic, M., Barkoczi, D., \& Katsikopoulos, K. (2018). Smaller crowds outperform larger crowds and individuals in realistic task conditions. Decision, 5(1), 1-15. doi: 10.1037/dec0000059

Goodin, R. E., \& Spiekermann, K. (2012, nov). Epistemic aspects of representative government. European Political Science Review, 4(03), 303-325. doi: 10.1017/ S1755773911000245

Kao, A. B., \& Couzin, I. D. (2014, apr). Decision accuracy in complex environments is often maximized by small group sizes. Proceedings of the Royal Society B: Biological Sciences, 281(1784), 20133305-20133305. doi: 10.1098/ rspb.2013.3305

Krogh, A., \& Vedelsby, J. (1995). Neural network ensembles, cross validation, and active learning. In G. Tesauro, D. S. Touretzky, \& T. K. Leen (Eds.), Advances in neural information processing systems (pp. 231-238). Cambridge, MA: MIT Press.

Kurvers, R. H. J. M., de Zoete, A., Bachman, S. L., Algra, P. R., \& Ostelo, R. (2018, apr). Combining independent decisions increases diagnostic accuracy of reading lumbosacral radiographs and magnetic resonance imaging. PLOS ONE, 13(4), e0194128. doi: 10.1371/journal.pone.0194128

Kurvers, R. H. J. M., Herzog, S. M., Hertwig, R., Krause, J., Carney, P. A., Bogart, A., .. Wolf, M. (2016, jul). Boosting medical diagnostics by pooling independent judgments. Proceedings of the National Academy of Sciences, 201601827. doi: 10.1073/pnas.1601827113

Larrick, R. P., \& Soll, J. B. (2006, jan). Intuitions about combining opinions: Misappreciation of the averaging principle. Management Science, 52(1), 111-127. doi: 10.1287/mnsc. 1050.0459

Macmillan, N. A., \& Creelman, C. D. (2005). Detection Theory: A User's Guide (2nd ed., Vol. 31) (No. 1). Mahwah, NJ: Lawrence Erlbaum. doi: 10.1017/ CBO9781107415324.004

Mannes, A. E., Soll, J. B., \& Larrick, R. P. (2014). The wisdom of select crowds. Journal of Personality and Social Psychology, 107(2), 276-299. doi: 10.1037/a0036677

Olsson, H., \& Loveday, J. (2015). A comparison of small crowd selection methods. In D. C. Noelle et al. (Eds.), Proceedings of the thirty-seventh annual conference of the cognitive science society (pp. 1769-1774). Austin, TX: Cognitive Science Society.

Page, S. E. (2007). The difference: How the power of diversity creates better groups, firms, schools, and societies. Princeton, NJ: Princeton University Press.

Reinhard, M.-A., Greifeneder, R., \& Scharmach, M. (2013). Unconscious processes improve lie detection. Journal of Personality and Social Psychology, 105(5), 721-739. doi:

\section{$10.1037 / \mathrm{a} 0034352$}

Rice, M. E., \& Harris, G. T. (1995). Violent recidivism: Assessing predictive validity. Journal of Consulting and Clinical Psychology, 63(5), 737-748. doi: 10.1037/0022 $-006 X .63 .5 .737$

Singh, J. P. (2013, jan). Predictive validity performance indicators in violence risk assessment: A methodological primer. Behavioral Sciences and the Law, 31(1), 8-22. doi: $10.1002 / \mathrm{bsl} .2052$

Sorkin, R., Hays, C., \& West, R. (2001). Signal-detection analysis of group decision making. Psychological Review, 108(1), 183-203. doi: 30.1037//0033-295X.

Spiekermann, K., \& Goodin, R. E. (2012). Courts of many minds. British Journal of Political Science, 42(3), 555571. doi: 10.1017/S000712341100041X

Sunstein, C. R. (2006). Infotopia: How many minds produce knowledge. New York: Oxford University Press.

Surowiecki, J. (2004). The wisdom of crowds. New York: Doubleday. 